\title{
Arrays of optical vortices formed by 'fork' holograms
}

\author{
Bekshaev A. Ya., Bekshaev A. S. and Mohammed K. A. \\ I. I. Mechnikov Odessa National University, \\ 2 Dvorianska Street, 65082 Odessa, Ukraine, bekshaev@onu.edu.ua
}

Received: 16.06 .2014

\begin{abstract}
Singular light beams with optical vortices (OVs) are often generated by means of thin binary gratings with groove bifurcation ('fork' holograms), which produce a set of diffracted beams with different OV charges. Though a single separate beam is usually employed and studied, here we consider a whole set of diffracted $\mathrm{OV}$ beams which, under certain conditions, are involved in efficient mutual interference to form a characteristic pattern where the ring-like structure of separate OV beams is replaced by a series of bright and dark lines between the adjacent diffraction orders. This pattern, which is well developed for high diffraction orders, reflects the main spatial properties of the diffracted beams as well as those of a 'fork' grating used for their generation. In particular, this confirms a theoretical model for the diffracted beams, a so-called Kummer beam model, and enables one to determine the sign and the absolute value of the phase singularity embedded in the hologram.
\end{abstract}

Keywords: optical vortex generation, 'fork' hologram, Kummer beam, interference

PACS: $42.25 . \mathrm{Fx}, 42.25 . \mathrm{Hz}, 42.40 . \mathrm{Eq}, 42.40 . \mathrm{Jv}, 42.50 . \mathrm{Tx}, 42.90 .+\mathrm{m}$

UDC: $537.871 .1+535.4+778.38$

Light beams with optical vortices (OVs), or screw wavefront dislocations, have been attracting a great attention of researchers for several decades [1-5]. Numerous researches and practical applications of OVs have given rise to diverse methods for their generation. A thin holographic grating with groove bifurcation (a 'fork') is one of the simplest and the most universal practical means designed for this purpose [6-10]. Usually, when an incident paraxial beam with regular (non-vortex) wavefront (e.g., a monochromatic Gaussian laser beam with a wavenumber $k$ ) intersects the central part of such a 'fork' grating (FG) along the normal to its plane, a set (or a 'fan') of diffracted beams with directions determined by the angles

$$
\theta_{n}=n \frac{2 \pi}{k d},
$$

which depend on the diffraction order $n$ and the grating period $d$, is formed behind the grating (see Fig. 1). The $n$ th-order diffracted beam carries the OV with the topological charge

$$
l_{n}=n q,
$$

where $q$ is a fixed topological charge of the phase singularity 'embedded' in the grating. In this report we restrict ourselves to the case of integer $q$, where the 'central' vertical groove is divided into $(|q|+1)$ branches (in particular, we have $q=1$ in Fig. 1).

In general practice, only one of the diffracted beams with a desired OV charge is used; all the other diffracted orders are filtered out and take no part in further manipulations. However, simultaneous observation and analysis of the multiple diffracted beams may provide some 
important information and give a unique possibility for analyzing the spatial structure of the separate diffracted beams. Besides, this can enable diagnosing the properties of the generating diffraction element.

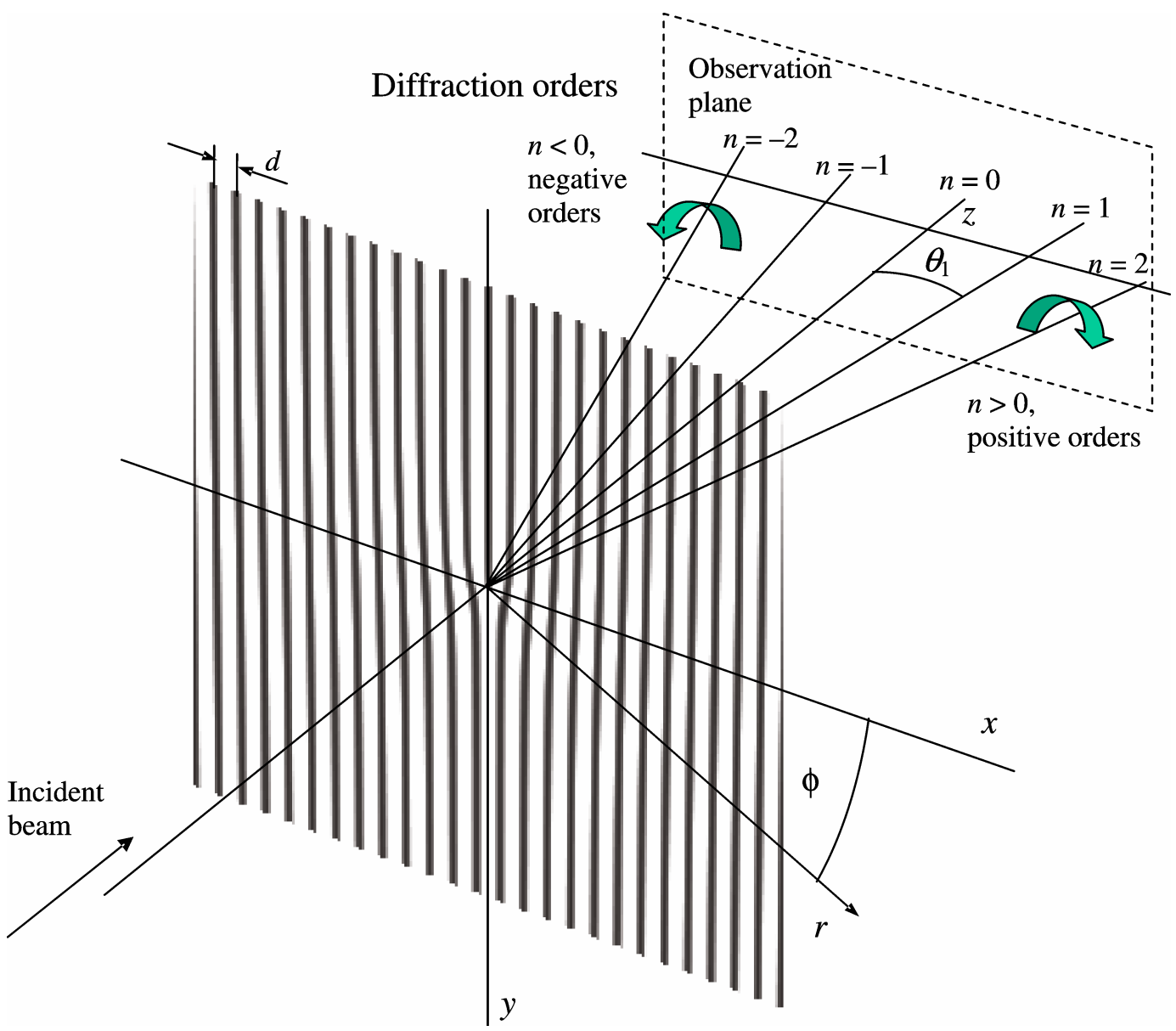

Fig. 1. Multiple OV beams produced by an FG. The Cartesian coordinate system $(x, y, z)$ is associated with the grating so that the $z$ axis is normal to the FG plane and intersects it exactly in the bifurcation point (the $F G$ 'centre'), while the $y$ axis is parallel to the grooves far from the centre. All of the axes of diffracted beams belong to the diffraction plane $x z$.

A characteristic pattern displayed in Fig. 2 can easily be observed when dealing with a 'simple' binary FG which has no special groove profiling aimed at removing some diffraction orders or, at least, at minimizing their numbers available. Normally it cannot be seen in the vicinity of the incident beam axis where the diffracted beams of the several first orders are concentrated, which are the most intense and so convenient for observation. But at the periphery of the diffracted 'fan', the diffracted beams start overlapping. This manifests itself in characteristic interference fringes that 'stretch' between the adjacent diffracted OV beams so that the expected ring-like beam spots are completely replaced by the quasiperiodic patterns of bright and dark strips (see Fig. 2).

Quite unexpectedly, we have not been able to find any description or interpretation of such patterns in the current literature. This is why the main purpose of this work is to explain the pattern and inspect how it can be used for investigating the spatial structure of the diffracted beams and for diagnosing the FG structure. 


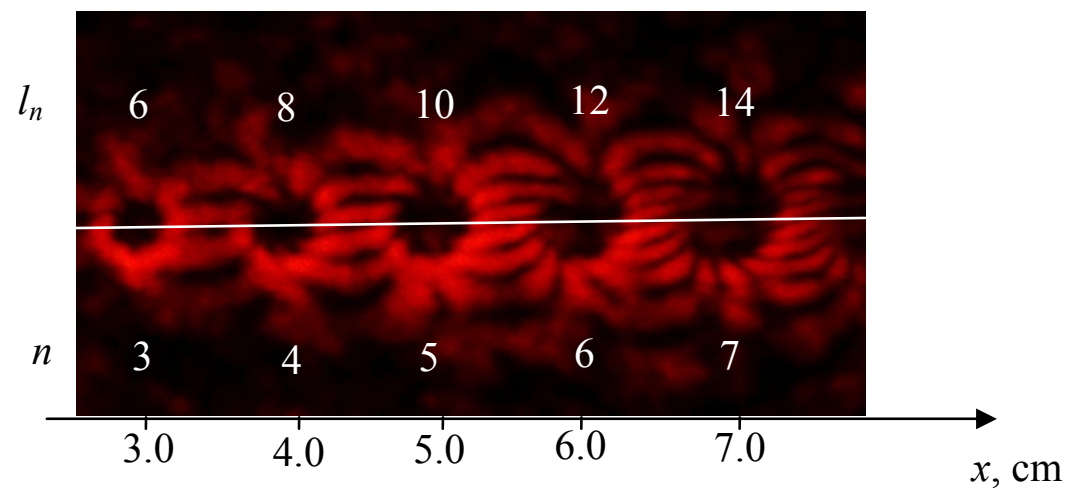

Fig. 2. Pattern of multiple OV beams formed after diffraction of a Gaussian laser beam with the waist radius $b_{0}=0.2 \mathrm{~mm}$ at the FG with $q=2$, as observed in the $3^{\text {rd }}-7^{\text {th }}$ diffracted orders on a screen $z=100 \mathrm{~cm}$ distanced from the FG. Expected topological charges of the generated OV beams are indicated above the OVs; figures below indicate the diffraction orders; distance $x$ is measured from the zero-order beam centre; white line is a trace of the diffraction plane. The other parameters of the experiment are as follows: $d=1 / 16 \mathrm{~mm}, k \approx 10^{5} \mathrm{~cm}^{-1}$ (He-Ne laser), and $\theta_{1}=0.01 \mathrm{rad}$.

We start with noticing that the spatial distribution of the complex amplitude of the $n$ th-order diffracted beam in the screen plane can be expressed in the form

$$
u_{n}(x, y, z)=U_{n}\left(r_{n}\right) \exp \left(i l_{n} \varphi_{n}\right) \exp \left[i \frac{k a_{n}}{z}\left(x-a_{n}\right)+i \frac{k}{2 z} a_{n}^{2}\right],
$$

where $a_{n}=n z \theta_{1}$ is the position of the $n^{\text {th }}$ diffracted beam axis on the screen (the condition $\theta_{1}<<1$ is implied). Here we have

$$
r_{n}(x, y, z)=\sqrt{\left(x-a_{n}\right)^{2}+y^{2}}, \exp \left[i \varphi_{n}(x, y, z)\right]=\frac{\left(x-a_{n}\right)+i \sigma y}{r_{n}},
$$

with $\sigma=\operatorname{sgn}(q)= \pm 1$ being the sign of the phase singularity embedded in the FG. The last exponential term in Eq. (3) expresses the additional phase of slightly inclined $n$ th-order beam in the observation plane normal to the nominal axis $z$ (see Fig. 1). Then the resulting observed field pattern may be expressed as

$$
I_{M, N}(x, y, z)=\left|\sum_{n=M}^{N} u_{n}(x, y, z)\right|^{2},
$$

where $M$ and $N$ are the minimal and maximal numbers of the diffraction orders whose influence cannot be neglected. Note that simultaneous replacements $x \rightarrow-x, n \rightarrow-n$ and $\sigma \rightarrow-\sigma$ do not modify Eq. (3) and, consequently, Eq. (5), so that the resulting field pattern is symmetric with respect to the $y$ axis. In full agreement with the experimental observation, this permits us to restrict our consideration to the positive $x$ only. For this reason, only positive diffraction orders, $n>0$, will be considered below.

In the most cases one can expect that the field pattern in the region $a_{n}<x<a_{n+1}$ may be evaluated via Eq. (5) by substituting $M=n$ and $N=n+1$, i.e. allowance for only two adjacent diffracted beams is satisfactory. However, Eq. (5) gives a possibility to take into account the influence of long-distanced members of the sequence of diffracted beams, besides of the nearest ones.

The function $U_{n}\left(r_{n}\right)$ describes the details of the diffracted beam shape, which depend on the model used for its characterization. In many cases, when the main attention is paid to the screw 
wavefront dislocation and the vortex properties of the FG-generated diffracted beam, it is suitable to describe it with a standard OV beam model supplied by the Laguerre-Gaussian (LG) mode [1, $3,4]$ :

$$
U_{n}\left(r_{n}\right) \equiv U_{n}^{L G}\left(r_{n}\right)=\left(\frac{r_{n}}{b}\right)^{\left|l_{n}\right|} \exp \left(-\frac{r_{n}^{2}}{2 b^{2}}\right) \exp \left[i k \frac{r_{n}^{2}}{2 R}-i\left(\left|l_{n}\right|+1\right) \chi\right],
$$

where the beam radius $b$, the wavefront curvature radius $R$ and the additional phase shift (Gouy phase) $\chi$ are conveniently fitted [11]. These are associated with the 'conventional' beam waist radius $b_{1}$ and the corresponding 'conditional' Rayleigh range $z_{R 1}=k b_{1}^{2}$ chosen so that Eq. (8) could supply the best fitting to the real diffracted OV beam:

$$
R(z)=\frac{z_{R 1}^{2}+z^{2}}{z}, b^{2}(z)=\frac{z_{R 1}^{2}+z^{2}}{k z_{R 1}}, \chi(z)=\arctan \left(\frac{z}{z_{R 1}}\right) .
$$

The LG approximation given by Eq. (6) can be useful while describing the spatial profile of a single diffracted beam, at least in its separate cross sections [3, 6, 7, 11, 12]; however, it becomes invalid when applied to the 'fan' of diffracted OV beams simultaneously produced by a single FG. Fig. 3 demonstrates that, even in the situation where the beams efficiently overlap, the interference pattern calculated via the LG representation is quite different from that observed in practice: the fringes are almost rectilinear and strictly localized inside the bright rings. Although the calculations illustrated in Fig. 3 have been performed for the values $M=3$ and $N=7$ in Eq. (5), exactly the same picture could be obtained by combining the interference patterns calculated for each pair of the nearest diffracted beams: this is a consequence of very rapid exponential decay of the LG beam intensity with growing $r_{n}$.

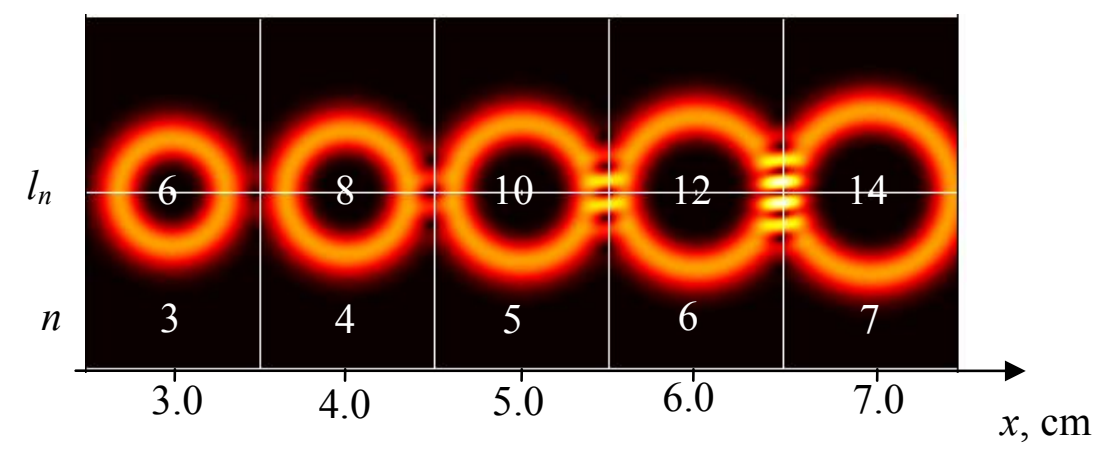

Fig. 3. Pattern of multiple OV beams formed by $F G(q=2)$ in the $3^{\text {rd }}-7^{\text {th }}$ diffracted orders under conditions corresponding to Fig. 2, as calculated with Eq. (5) ( $\mathrm{M}=3$ and $N=7$ ) for the LG model given by Eqs. (6) and (7). 'Conventional' waist radius is $b_{1}=0.08 \mathrm{~mm}$, vertical lines correspond to the middle distance between the axes of diffracted beams, and horizontal line is a trace of the diffraction plane (see Fig. 1). Topological charges are indicated near the OV centers and corresponding diffraction orders are indicated as in Fig. 2, and distance $\mathrm{x}$ is measured from the zero-order beam centre.

In this situation, one should resort to a more accurate model of the diffracted OV beam. As known for the case of a simple binary FG, the most adequate is a model of Kummer (or hypergeometric Gaussian) beam $[10,11,13]$ :

$$
U_{n}\left(r_{n}\right) \equiv U_{n}^{K}\left(r_{n}\right)=(-i)^{\left|l_{n}\right|+1} \exp \left(\frac{i k}{2 z} r_{n}^{2}\right) \frac{z_{R}}{z-i z_{R}} \sqrt{A_{n}} \exp \left(-A_{n}\right)\left[I_{\frac{\left|l_{n}\right|-1}{2}}\left(A_{n}\right)-I_{\frac{\left|l_{n}\right|+1}{2}}\left(A_{n}\right)\right],
$$

where 


$$
z_{R}=k b_{0}^{2}
$$

is the Rayleigh range of the incident Gaussian beam waist of which is supposed to coincide with the FG plane (with $b_{0}$ being the waist beam radius at the level $e^{-1}$ of the maximum intensity), and

$$
A_{n}=\left(\frac{k r_{n}}{z}\right)^{2} \frac{b^{2}}{4\left(1-i z_{R} / z\right)} .
$$

The results of calculations based on Eqs. (7)-(9) and (5) for $M=3$ and $N=7$ are presented in Fig. 4, which should be compared to Fig. 2. Since the diffracted beams with $n<3$ and $n>7$ are not taken into account, Fig. 4 is expected to represent the correct results only in the region $3 \mathrm{~cm}<x<7 \mathrm{~cm}$, including the $4^{\text {th }}-, 5^{\text {th }}$ - and $6^{\text {th }}$-order diffracted beams. Confronting this pattern with the relevant area shown in Fig. 2, we conclude that the calculated pattern is qualitatively similar to the experimental one and so the Kummer beam model of the diffracted beams indeed explains the actual observations.

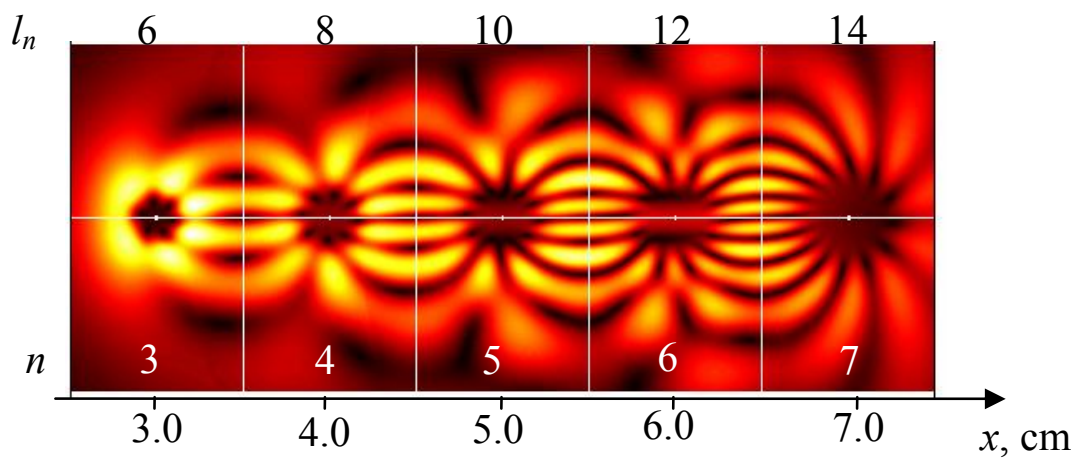

Fig. 4. Pattern of multiple OV beams formed by FG $(q=2)$ in the $3^{\text {rd }}-7^{\text {th }}$ diffracted orders under conditions corresponding to Fig. 2, as calculated for the Kummer beam model given by Eqs. (7)-(9) with $b_{0}=0.2 \mathrm{~mm}$. Topological charges, diffraction orders, and distances $x$ and $z$ are the same as in Fig. 2 and Fig. 3. The meaning of white lines is the same as in Fig. 3.

Eqs. (3), (7) and (5) describe fairly well the field structure observed, though they are not suitable for analytical quantitative analysis. To make the latter more feasible, we remark that the main details of the interference pattern, e.g. the locations of the dark and bright lines, are determined by the phase of the field given by Eq. (7), whereas the field amplitude specifies only their relative brightnesses. Therefore, we consider the 'purely phase' part of Eq. (7) which can be treated as a version of a more general Eq. (3), with

$$
U_{n}\left(r_{n}\right) \equiv U_{n}^{P}\left(r_{n}\right)=(-i)^{l_{n} \mid+1} \exp \left(\frac{i k}{2 z} r_{n}^{2}\right) .
$$

In this case one can easily find the 'intensity distribution', taking only the two nearest diffraction orders into account:

$$
I_{n, n+1}(x, y, z)=2\left\{1+\cos \left[\left(l_{n+1} \varphi_{n+1}-l_{n} \varphi_{n}\right)-\frac{\pi}{2}\left(\left|l_{n+1}\right|-\left|l_{n}\right|\right)\right]\right\},
$$

where Eqs. (4) have been employed. Just at the middle distance between the axes of the $n$th and $(n+1)^{\text {th }}$ diffracted beams with the topological charges $l_{n}$ and $l_{n+1}$,

$$
x=\frac{a_{n+1}+a_{n}}{2},
$$


the angular parameters $\varphi_{n}$ and $\varphi_{n+1}$ in Eq. (11) are determined by the relations

$$
\varphi_{n} \equiv \varphi_{n}^{m}=\arctan \frac{2 y}{a_{n+1}-a_{n}}, \varphi_{n+1}=\pi-\varphi_{n}^{m} .
$$

In view of Eq. (2), we have $l_{n+1} \varphi_{n+1}^{m}-l_{n} \varphi_{n}^{m}=q(n+1) \pi-q(2 n+1) \varphi_{n}^{m}$ and so we get

$$
\begin{aligned}
I_{n, n+1} & =2+2 \cos \left[\left(l_{n+1}+l_{n}\right) \varphi_{n}^{m}-\frac{\pi}{2}\left(\left|l_{n+1}\right|+\left|l_{n}\right|\right)\right] \\
& =2+2 \cos \left[\left(\left|l_{n+1}\right|+\left|l_{n}\right|\right)\left(\varphi_{n}^{m}-\frac{\pi}{2} \operatorname{sgn}\left(l_{n}\right)\right)\right] \\
& =2+2 \cos \left[|q|(2 n+1)\left(\varphi_{n}^{m}-\frac{\pi}{2} \operatorname{sgn}(q)\right)\right] \\
& =2+2\left\{\begin{array}{c}
(-1)^{q / 2} \cos \left[|q|(2 n+1) \varphi_{n}^{m}\right], \text { if } q \text { is even; } \\
(-1)^{n+(q-1) / 2} \sin \left[|q|(2 n+1) \varphi_{n}^{m}\right], \text { if } q \text { is odd }
\end{array}\right.
\end{aligned}
$$

For a known $n$, this gives the absolute value and the sign of $q$. The absolute value $|q|$ can be determined from the number of intensity zeros or maximums situated along the vertical line given by Eq. (12). The number of zeros is

$$
Z=\left\lfloor|q| \frac{2 n+1}{2}\right\rfloor,
$$

where $\lfloor\ldots\rfloor$ implies the integer part of a number, whereas the number of the maximums is the same for odd $q$ and equals to $Z-1$ for even $q$.

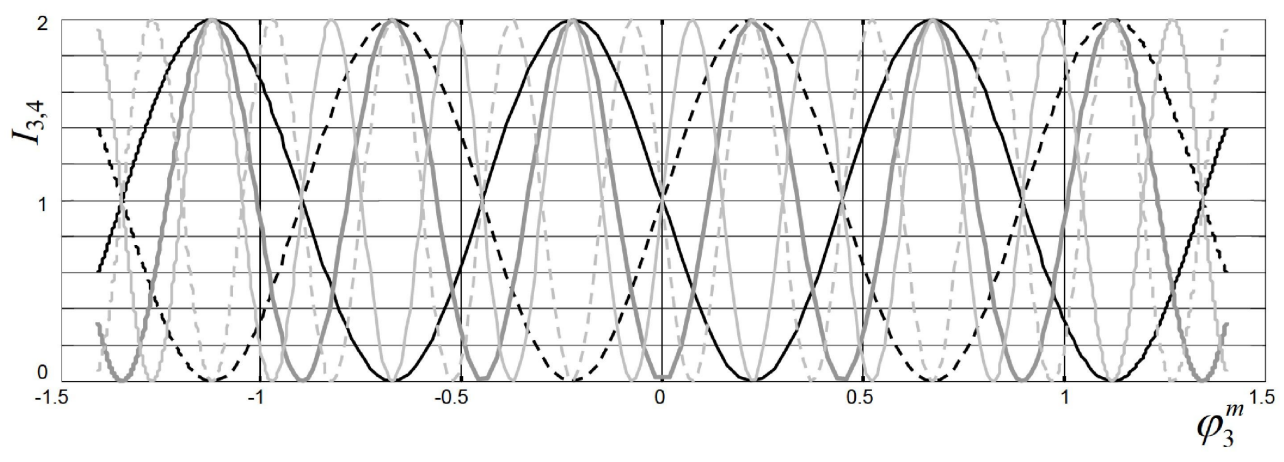

Fig. 5. Intensity distributions along the middle line specified by Eq. (12) between the diffracted beams $n=3$ and $n=4$. Solid lines correspond to positive $q$ and dashed lines to negative $q$. Black lines correspond to $|q|=1$, grey to $|q|=2$ and light grey to $|q|=3$. Solid and dashed grey lines coincide with each other.

The above situation is illustrated by Fig. 5 . The $|q|$ value can be readily determined from the number of minima or maxima observed, using Eq. (15). In addition, the cases of positive and negative $q$ differ in a distinct way: the positions of the maxima for $q>0$ turn into those of the minima for $q<0$, and vice versa. This does not work in case of even $q$ : the solid and dashed grey lines in Fig. 5 coincide and, therefore, the sign of even $q$ cannot be determined from the positions of intensity minima and maxima in the middle line given by Eq. (12). However, the situations with the positive and negative $q$ are rather different at the other points located between $x=a_{n}$ and $x=a_{n+1}$ : a comparison of Figs. 2, 3, 4 and 6 shows that the sign of $q$ can readily be identified via a slight inclination of the interference fringes near the $x$ axis. Due to combined action of the screw 
and spherical components of wavefronts of the diffracted orders, the interference lines are not parallel to the $x$ axis: they go slightly 'up' (or 'down') for the positive (or negative) $q$ with increasing $x$. Of course, this rule is also valid for the odd $q$, and, again, it can be used for detecting the $q$ sign. The latter solution may appear to be more suitable in practice than tracing alteration of the minima and maxima along the middle line defined by Eq. (12).

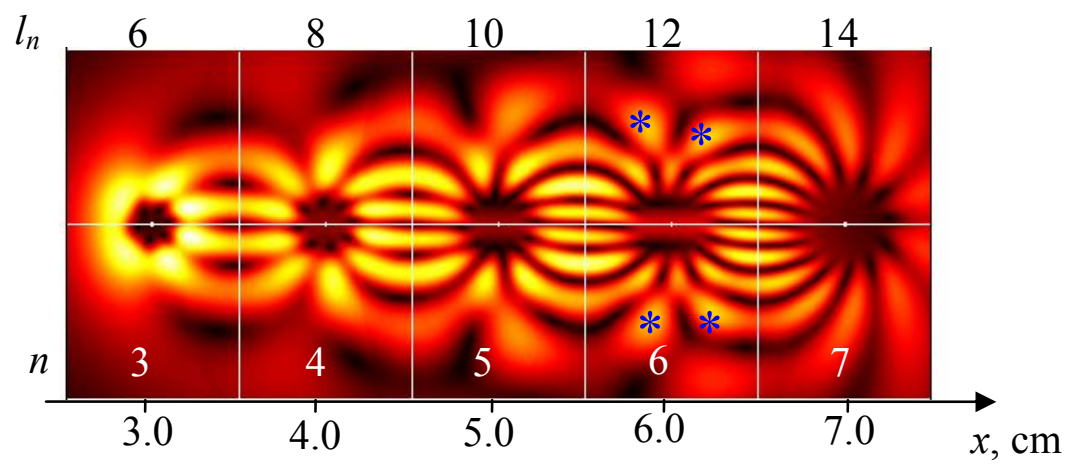

Fig. 6. Pattern of multiple OV beams formed by FG $(q=-2)$ in the $3^{\text {rd }}-7^{\text {th }}$ diffracted orders. The other conditions are the same as in Fig. 4.

Additional helpful rules can be seen from incremental growth in the numbers of interference fringes occurring with increasing diffraction order. According to Eq. (15), the total number of zeros or maxima for consecutive orders $n$ and $n+1$ increases by $|q|$. Under conditions corresponding to Fig. 4 and Fig. 6 for the lines $x=3.5$ (between the $3^{\text {rd }}$ and $4^{\text {th }}$ orders, $n=3$ ), 4.5 $(n=4)$ and $5.5(n=5), \ldots$, the number of zeros amounts respectively to $7,9,11, \ldots$, i.e. the increment is $2=|q|$. Likewise, in Fig. 7 this increment expectedly equals to 1 and in Fig. 8 it is 3 .

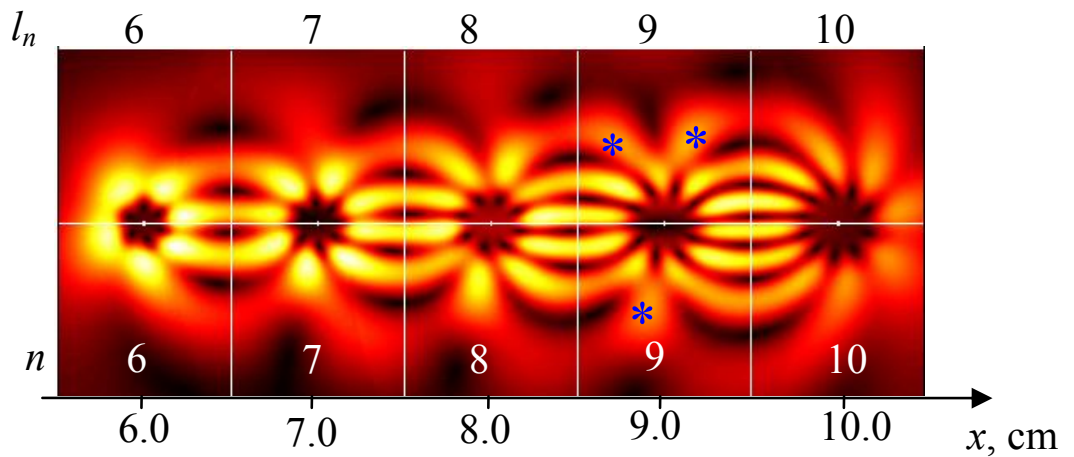

Fig. 7. Pattern of multiple OV beams formed by FG $(q=1)$ in the $6^{\text {th }}-10^{\text {th }}$ diffracted orders, as calculated for the Kummer beam model given by Eqs. (7)-(9) with $b_{0}=0.2 \mathrm{~mm}$. Distance $z$ is the same as in Figs. $2-4$ and 6 , and distance $x$ is measured from the zero-order beam axis. The meaning of white lines is the same as in Fig. 3 .

The regularities discussed above can be used, e.g., for express diagnostics of the FGs, if one needs to learn on the topological charge $q$ of the phase singularity embedded. However, detection of some intensity minima or maxima located far from the $x$ axis can be difficult in practice because of low diffracted beam intensities at large $y$. For example, following from Eq. (15) the number of zeros along the vertical lines $x=3.5 \mathrm{~cm}(n=3)$ and $x=4.5 \mathrm{~cm}(n=4)$ in Fig. 4 and Fig. $6(q=2)$ should be equal to 7 and 9 , while only 5 and 7 minima are really seen: the intensity minima situated at high $|y|$ are hardly discernible in practice because of the low local intensity and inevitable noise contamination. In such cases, observation of the interference pattern within a 
limited vertical segment can be purposeful. If one takes only the brightest interference lines into account, their numbers in Fig. 2 are 3, 5 and 7 (between the $3^{\text {rd }}$ and $4^{\text {th }}$, the $4^{\text {th }}$ and $5^{\text {th }}$, and the $5^{\text {th }}$ and $6^{\text {th }}$ orders); in Fig. 4 and Fig. 6 one sees 4, 6 and 8 lines, thus clearly testifying the value $|q|=2$. This criterion seems to be the most stable against practically inevitable noises and distortions: in practical experiments, due to the presence of many orders, it is usually not difficult to find several of those orders for which the incremental growth in the fringe numbers can be reliably identified.

In more accurate measurements, the positions of the bright lines near the $x$ axis can also be helpful. According to Eq. (14), the regularities are different for the even and odd $|q|$. In case of even $|q|$, the interference pattern for every $n$ reveals an extremum at $y=0$ : a minimum for $|q|=2$, $6,10, \ldots$ and a maximum for $|q|=4,8,12, \ldots$ (cf. Fig. 1, Fig. 4 and Fig. 6). In case of odd $|q|$, the maxima and minima are situated just above or just below the horizontal axis $y=0$, and, for $|q|=1$, $5,9, \ldots$, the intensity above the $x$ axis is maximal at even $n$ and minimal at odd $n$ (cf. the interference lines between the diffracted beams of the $6^{\text {th }}$ and $7^{\text {th }}, 7^{\text {th }}$ and $8^{\text {th }}, 8^{\text {th }}$ and $9^{\text {th }}$, and $9^{\text {th }}$ and $10^{\text {th }}$ orders in Fig. 7). For $|q|=3,7,11, \ldots$, the vertical intensity distribution along the middle line given by Eq. (12) shows the opposite behaviour: one can see the minima above the $x$ axis at even $n$ and the maxima at odd $n$ (cf. the interference lines between the diffracted beams of the $2^{\text {nd }}$ and $3^{\text {rd }}$, $3^{\text {rd }}$ and $4^{\text {th }}, 4^{\text {th }}$ and $5^{\text {th }}$, and $5^{\text {th }}$ and $6^{\text {th }}$ orders in Fig. 8).

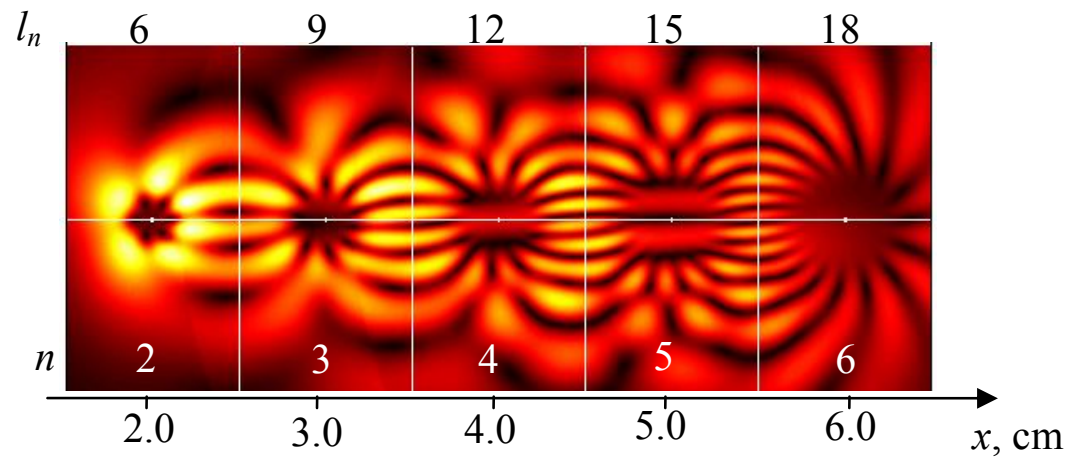

Fig. 8. Pattern of multiple OV beams formed by FG $(q=3)$ in the $2^{\text {nd }}-6^{\text {th }}$ diffraction orders, as calculated for the Kummer beam model given by Eqs. (7)-(9) with $b_{0}=0.2 \mathrm{~mm}$. Distance $z$ is the same as in Fig. 7 and distance $x$ is measured from the zero-order beam axis. The meaning of white lines is the same as in Fig. 3 .

The other possible approaches to diagnosing the FGs can employ the apparent fact visible in all of the above figures: due to superposition of the beams of different diffraction orders, an expected ring-like structure in each order is 'fractioned' into a 'necklace' of bright spots, whose number systematically increases with increasing $n$ and $|q|$. However, the considered examples show no distinct quantitative regularity in this increase: while the rings corresponding to $n=4,5$ and 6 in Fig. 4 and Fig. 6 are split respectively into 8,10 and 12 spots, the rings $n=8$ and $n=9$ in Fig. 7 are both split into 10 spots. In the situation observed in Fig. 8, the rings of the orders $n=3$, 4 and 5 are decomposed into 10,14 and 18 spots. Here a sort of regularity seems to be restored, though the increment value 4 is not quite understandable. Besides, the experimental identification of the separate spots within the presumed rings is sometimes difficult. For instance, in Fig. 6 and Fig. 7 there are 'doubtful' bright spots within the expected $6^{\text {th }}$ - and $9^{\text {th }}$-order 'rings' which are marked by asterisks. They are positioned at larger distances from the beam axis than the other ones, for which reason they have not been taken into account when reckoning the number of the ring 'fragments' a few lines above. However, inevitable distortions of the theoretical pattern in real 
experiments can make these maxima visually equivalent to the others so that calculations of the number of bright spots may be ambiguous. Probably, for this reason the numbers of the bright spots in the 'necklaces' seen in Fig. 2 differ from the theoretical predictions illustrated in Fig. 4 and Fig. 6.

\section{References}

1. Allen L, Padgett M J and Babiker M, 1999. Orbital angular momentum of light. Prog. Opt. 39: 291-372.

2. Berry M, 1998. Paraxial beams of spinning light. Proc. SPIE. 3487: 6-11.

3. Soskin M S and Vasnetsov M V, 2001. Singular optics. Prog. Opt. 42: 219-276.

4. Allen L, Barnett S M and Padgett M J. Optical angular momentum. Bristol: Institute of Physics Publishing (2003).

5. Bekshaev A Ya, Soskin MS and Vasnetsov MV. Paraxial light beams with angular momentum. N. Y.: Nova Science Publishers (2008).

6. Bazhenov V Yu, Vasnetsov M V and Soskin M S, 1990. Laser beams with screw dislocations in their wavefronts. JETP Lett. 52: 429-431.

7. Heckenberg N R, McDuff R, Smith C P, Rubinstein-Dunlop H and Wegener M J, 1992. Laser beams with phase singularities. Opt. Quant. Electron. 24: S951-S962.

8. Basistiy I V, Soskin M S and Vasnetsov M V, 1995. Optical wavefront dislocations and their properties. Opt. Commun. 119: 604-612.

9. Rozas D, Law C T and Swartzlander G A, Jr, 1997. Propagation dynamics of optical vortices. J. Opt. Soc. Amer. B. 14: 3054-3065.

10. Sacks Z S, Rozas D and Swartzlander G A, Jr, 1998. Holographic formation of optical-vortex filaments. J. Opt. Soc. Amer. B. 15: 2226-2234.

11. Bekshaev A Ya and Karamoch A I, 2008. Spatial characteristics of vortex light beams produced by diffraction gratings with embedded phase singularity. Opt. Commun. 281: 13661374.

12. Sviridova S V and Bekshaev A Ya, 2011. Transformation of the optical vortex light beams in holographic elements with embedded phase singularities. Proc. SPIE. 8338: 83380A.

13. Karimi E, Zito G, Piccirillo B, Marrucci L and Santamato E, 2007. Hypergeometric-Gaussian modes. Opt. Lett. 32: 3053-3055.

Bekshaev A.Ya., Bekshaev A.S. and Mohammed K.A. 2014. Arrays of optical vortices formed by 'fork' holograms. Ukr.J.Phys.Opt. 15: 123 - 131.

Анотація. Сингулярні оптичні пучки, що переносять оптичні вихори, здебільшого генерують за допомогою тонких бінарних траток з біфуркацією штрихів ("вилкоподібні" голограми), які продукують набір дифрагованих пучків з вихорами різних зарядів. Зазвичай використовують і досліджують лише один окремо взятий пучок. У иій роботі розглянуто весь набір дифрагованих вихрових пучків, які за певних умов взаємно інтерферують $i$ формують характерні смуги, для яких кільчева структура окремих вихрових пучків заміняється серією яскравих і темних ліній між сусідніми дифракційними порядками. Ці смуги, добре розвинуті для високих дифракиійних порядків, виявляють основні просторові властивості дифрагованих пучків і вилкоподібної гратки, використаної для їхньої генерації. Зокрема, че підтверджує теоретичну модель дифрагованих пучків (модель пучків Куммера) $i$ дає змогу визначити знак $i$ абсолютну величину фазової сингулярності, вбудованої в голограму. 